\title{
Scattering Theory for Systems with Different Spatial Asymptotics on the Left and Right ${ }^{\star}$
}

\author{
E. B. Davies ${ }^{\star \star}$ and B. Simon ${ }^{\star \star \star}$ \\ Department of Mathematics, Princeton University, Princeton, New Jersey, USA
}

\begin{abstract}
We discuss the existence and completeness of scattering for onedimensional systems with different spatial asymptotics at $\pm \infty$, for example $-\frac{d^{2}}{d x^{2}}+V(x)$ where $V(x)=0$ (resp. $\left.\sin x\right)$ if $x<0$ (resp. $x>0$ ). We then extend our results to higher dimensional systems periodic, except for a localised impurity, in all but one space dimension. A new method, "the twisting trick", is presented for proving the absence of singular continuous spectrum, and some independent applications of this trick are given in an appendix.
\end{abstract}

\section{§1. Introduction}

This paper began with a question posed to us by G. Papanicolaou: Consider a potential $W$ on $\mathbb{R}$ which is periodic and let $V$ be the potential which is zero on $(-\infty, 0)$ and equal to $W$ on $(0, \infty)$, so that $-\frac{d^{2}}{d x^{2}}+V$ is a quantum Hamiltonian for a "half-line of solid". It is well-known $[19,30,31,37]$ that the spectrum of $-\frac{d^{2}}{d x^{2}}+W$ is a series of bands $\left[\alpha_{1}, \beta_{1}\right] \cup\left[\alpha_{2}, \beta_{2}\right] \cup \ldots$ where in general $\beta_{i}<\alpha_{i+1}$. Suppose that one sends an electron into the half-solid system from the left with energies strictly in some gap $\left(\beta_{i}, \alpha_{i+1}\right)$. How can one prove total reflection from the half-solid?

This is one of several scattering problems on the line involving different spatial asymptotics as $x \rightarrow \pm \infty$. Here are two others: (i) (Hard wall). Suppose that $V(x) \rightarrow 0$ faster than $|x|^{-1-\varepsilon}$ as $x \rightarrow-\infty$ but that $V(x) \rightarrow \infty$ as $x \rightarrow+\infty$. One expects total reflection at all energies. (ii) (Klein paradox). Suppose that $|x|^{1+\varepsilon}\left(V(x)-a_{ \pm}\right) \rightarrow 0$ as $x \rightarrow \pm \infty$ but with $a_{+} \neq a_{-}$. If $a_{-}<a_{+}$one expects total

\footnotetext{
${ }^{\star}$ Research supported by NSF grant MPS-75-11864

${ }^{\star \star}$ On leave from Mathematical Institute, Oxford OX1 3JP, England

$\star \star \star A l s o$ at Department of Physics, Princeton University
} 
reflection for energies in $\left(a_{-}, a_{+}\right)$but a "normal" scattering theory for energies above $a_{+}$. Among our results are proofs of existence and completeness of scattering states for all the above problems.

We have called (ii) above the Klein paradox because if $\frac{d^{2}}{d x^{2}}$ is replaced by $\left(-i \alpha \frac{d}{d x}+\beta\right)$ where $\alpha$ is the two by two matrix $\left(\begin{array}{ll}0 & 1 \\ 1 & 0\end{array}\right)$ and $\beta=\left(\begin{array}{rr}1 & 0 \\ 0 & -1\end{array}\right)$ then one has the famous problem analysed by Klein [26]: if $a_{+}-a_{-}>2 m$ then "more is reflected than went in". In their analysis of this problem Ruijenaars and Bongaarts [40] supposed that $V(x)=a_{ \pm}$if $\pm x>R$ for some $R$. While we shall not give any details, it is easy to see that our methods can be adapted to the Dirac case; thus our results subsume those of [40] in both the Dirac and Schrödinger case. We also note that Alsholm and Kato have existence results for problem (ii), [3].

Our solution of these problems is motivated in part by work of several authors $[10,14,32,41]$ on the effect that severe local singularities can have in scattering theory. In particular we rely heavily on technical devices from Combescure and Ginibre [10].

In one dimension it would be easier to use the Deift and Simon method [14], which we mention since it "explains" why these problems are quite easy. Deift and Simon decoupled finite singularities from the questions of existence and completeness by surrounding the singularity with a Dirichlet surface. In one dimension we need only note that adding a Dirichlet boundary at the origin decouples the left and right to conclude that the method of $[10,14]$ should allow control of existence and completeness for the problems mentioned above.

In the above problems there are natural comparison dynamics for forming wave operators, albeit different comparison dynamics on the left and right. It is nevertheless illuminating to make the analysis in two steps, the first of which makes no mention of any comparison dynamics. In Section 3 we consider operators $H=-\frac{d^{2}}{d x^{2}}+V$ on $L^{2}(\mathbb{R})$ for an extremely large class of $V$, including all $V$ which are bounded below irrespective of how wild their behaviour is at infinity. We will show that the projection $P_{\text {ac }}$ onto the absolutely continuous subspace of $H$ has two natural decompositions

$$
P_{\mathrm{ac}}=P_{l}^{+}+P_{r}^{+}=P_{l}^{-}+P_{r}^{-}
$$

where $\operatorname{Ran} P_{l}^{ \pm}\left(\right.$resp. $\left.\operatorname{Ran} P_{r}^{ \pm}\right)$consist of states which move towards $-\infty($ resp. $+\infty)$ as $t \rightarrow \mp \infty$. In cases where there are natural comparison Hamiltonians $H_{l}\left(\right.$ resp. $\left.H_{r}\right)$, which can be different on the left and right, we shall show that for $\phi \in \operatorname{Ran} P_{l}^{ \pm}$there is an $\eta_{ \pm}$such that

$$
\lim _{t \rightarrow \mp \infty}\left\|e^{-i t H} \phi-e^{-i t H_{1}} \eta_{ \pm}\right\|=0
$$

with a similar result on the right. This will provide a complete analysis of the basic scattering questions for the problems mentioned at the start of the section. Even in cases where there are no comparison Hamiltonians, one can still obtain some scattering results. We shall show in Section 4 that there is a function $R(E)$ 
defined in terms of $P_{l}^{ \pm}$alone, and which equals $|r(\sqrt{ } E)|^{2}$ whenever there is a natural comparison dynamics. We discuss the relationship of this result with other geometric approaches to scattering theory and also study the class of "homogeneous" potentials, defined as those for which $P_{l}^{+}=P_{r}^{-}$.

The analogue of $P_{l, r}^{ \pm}$in higher dimensions would be two projection-valued measures $P^{ \pm}(E)$ on the Sphere $S^{\nu-1}$ such that if $\phi \in \operatorname{Ran} P^{ \pm}(E)$ then as $t \rightarrow \mp \infty$, $e^{-i t H} \phi$ lies asymptotically in the cone $\left\{x \in \mathbb{R}^{v}: x /|x| \in E\right\}$. When the ordinary wave operators (or most types of modified wave operators) exist and are complete, one can prove the existence of such projection-valued measures, with $P^{ \pm}\left(S^{\nu-1}\right)=$ $P_{\mathrm{ac}}$. Even in multiparticle systems, when one has asymptotic completeness such a decomposition exists. The distinction between multichannel systems and the one-channel system is that in the latter case $P^{ \pm}(E)$ is absolutely continuous with respect to the usual measure on $S^{v-1}$ and in the former case it is not. However, we do not see an a priori method of proving the existence of $P^{ \pm}(E)$ in general multichannel systems or even in one-channel systems in dimension $v>1$. This is unfortunate since one can use the ideas in Sections 3 and 4 to replace the usual modified wave operators of long range scattering in one dimension.

There is a special but physically interesting class of systems in higher dimension which can be analysed by the methods herein, namely those systems which are periodic in all but one dimension. This includes in particular the multi-dimensional analogue of the half-solid. The key to the analysis is borrowed from Davies [11], whose results are subsumed by those in Sections 5 and 6. One uses the symmetry to realise the Hamiltonian as a direct integral of operators each of which can be viewed as a Schrödinger operator on $L^{2}(C)$, where $C$ is a one-dimensional cylinder whose $(v-1)$ dimensional base is a basic cell for the lattice of periods. As the parameter in the direct integral changes, so do the boundary conditions on $\partial C$. Since we have a direct integral of "essentially one-dimensional" systems, we are able to extend the ideas of Sections 2, 3 and 4, using some technicalities from [10].

If we denote the direct integral decomposition above by $H=\int^{\oplus} H(\theta) d \theta$ then the scattering states constructed in Section 5 correspond to direct integrals of states in $\operatorname{Ran} P_{\mathrm{ac}}(H(\theta)$ ). It can happen (indeed for systems periodic in all directions it does happen) that $\int^{\oplus} \operatorname{Ran} P_{\mathrm{ac}}(H(\theta)) d \theta$ is a strictly proper subspace of $\operatorname{Ran} P_{\mathrm{ac}}(H)$. In Section 6 we show that a direct integral of eigenstates of $H(\theta)$ can lie in $\operatorname{Ran} P_{\mathrm{ac}}(H)$, and that such states have an interpretation as "surface states". The natural spectral theory requirement that $\sigma_{\text {sing }}(H(\theta))=\varnothing$ for all $\theta$ then has the important physical consequence that any state in $\operatorname{Ran} P_{\mathrm{ac}}(H)$ is a sum of a surface state and a scattering state. Even in the half-solid case this spectral requirement does not seem to be susceptible to any of the usual methods of eliminating singular continuous spectrum. We therefore develop a new method, which we call the "twisting trick", and which allows us to embed $H(\theta)$ in a problem which can be treated by a limiting absorption principle. With these results we are able to analyse scattering from a "surface impurity" in Section 6.

The twisting trick is certainly the most significant new technical method in the paper. It has wider applicability than to the systems so far discussed. We examine some of these in Appendix 1. Included there is a proof that the Dirichlet 
Laplacian exterior to an arbitrary closed bounded set in $\mathbb{R}^{v}$ has empty singular continuous spectrum.

\section{§2. Asymptotic Projections: Some Abstract Nonsense}

Definition. Let $A$ be a self-adjoint operator on a Hilbert space $\mathscr{H}$ and let $J$ be a bounded operator. $J$ is called an asymptotic projection for $A$ if and only if

$$
S-\lim e_{t \rightarrow \pm \infty}^{i A t} J e^{-i A t} \equiv P_{\mp}(J ; A)
$$

exists and is an orthogonal projection.

Proposition 2.1. If $J_{0}$ is a bounded operator such that

(i) $\left[A, J_{0}\right]$ is trace class

(ii) $\left(J_{0}^{2}-J_{0}\right)$ and $\left(J_{0}^{*}-J_{0}\right)$ are compact then $J=J_{0} P_{\mathrm{ac}}(A)$ is an asymptotic projection for $A$.

Proof. The existence of $P(J ; A)$ under hypothesis (1) is a standard part of the Kato-Birman theory (see $[33,36]$ ). Moreover, since $e^{-i s A}$ commutes with $P(J ; A)$ it follows that

$$
P(J ; A)=\lim e^{i A t} P_{\mathrm{ac}}(A) J P_{\mathrm{ac}}(A) e^{-i A t}
$$

so that

$$
P^{*}(J ; A)-P(J ; A)=\lim e^{i A t} P_{\mathrm{ac}}(A)\left(J^{*}-J\right) P_{\mathrm{ac}}(A) e^{-i A t} .
$$

The right hand side vanishes since the compactness of $\left(J^{*}-J\right)$ implies that

$$
\underset{s \rightarrow \pm \infty}{s-\lim }\left(J^{*}-J\right) P_{\mathrm{ac}}(A) e^{-i A t}=0 .
$$

Similarly

$$
\begin{aligned}
P(J ; A)^{2}= & \lim e^{i A t} J_{0} e^{-i A t} P(J ; A) \\
= & \lim \left[e^{i A t} J_{0} e^{-i A t} P_{\mathrm{ac}}(A)\right. \\
& +e^{i A t}\left(J_{0}^{2}-J_{0}\right) e^{-i A t} P_{\mathrm{ac}}(A) \\
& \left.+e^{i A t} J_{0} e^{-i A t}\left(P(J ; A)-e^{i A t} J_{0} e^{-i A t} P_{\mathrm{ac}}(A)\right)\right] \\
= & P(J ; A) .
\end{aligned}
$$

Remark. Condition (ii) can be replaced by the hypothesis that $\left(J_{0}^{2}-J_{0}\right) E_{I}(A)$ and $\left(J_{0}^{*}-J_{0}\right) E_{I}(A)$ are compact for each bounded interval $I$, where $E_{I}(A)$ is the relevant spectral projection of $A$. Similarly (i) can be replaced by a variety of conditions, for example $J_{0} Q(A) \subseteq Q(A)$ and $E_{I}(A)\left[J_{0}, A\right] E_{I}(A)$ trace class, or the single condition that $\left[(A+c)^{-n}, J_{0}\right]$ is trace class for some $n$ (see [36]). Below we shall use Proposition 2.1 even under these alternative hypotheses.

Proposition 2.2. Let $J$ be an asymptotic projection for $A$. Then

(i) $\phi \perp \operatorname{Ran} P_{ \pm}(J ; A)$ if and only if $\left\|J e^{-i A t} \phi\right\| \rightarrow 0$ as $t \rightarrow \mp \infty$,

(ii) $\phi \in \operatorname{Ran} P_{ \pm}(J ; A)$ if and only if $\left\|(1-J) e^{-i A t} \phi\right\| \rightarrow 0$ as $t \rightarrow \mp \infty$. 
Proof. (ii) follows from (i) if we note that

$$
P_{ \pm}(1-J ; A)=1-P_{ \pm}(J ; A) .
$$

(i) holds since

$$
\lim _{t \rightarrow \mp \infty}\left\|J e^{-i A t} \phi\right\|=\left\|P_{ \pm}(J ; A) \phi\right\| \text {. }
$$

Proposition 2.3. Suppose that $J_{0}$ is a bounded operator such that $J_{0} P_{\mathrm{ac}}(A)$ is an asymptotic projection for $A$ and $J_{0}^{*} P_{\mathrm{ac}}(B)$ is an asymptotic projection for $B$. Suppose moreover that

$$
\begin{aligned}
& s-\lim e^{i t B} J_{0} e^{-i t A} P_{\mathrm{ac}}(A) \equiv \Omega^{ \pm}\left(B, A ; J_{0}\right) \\
& t \rightarrow \mp \infty
\end{aligned}
$$

and

$$
\lim _{t \rightarrow \mp \infty} e^{i t A} J_{0}^{*} e^{-i t B} P_{\mathrm{ac}}(B)
$$

exist. Then $\Omega^{ \pm}$is a partial isometry with initial space $\operatorname{Ran} P_{ \pm}\left(J_{0} P_{\mathrm{ac}}(A) ; A\right)$ and final space $\operatorname{Ran} P_{ \pm}\left(J_{0}^{*} P_{\mathrm{ac}}(B) ; B\right)$. Moreover if $\phi \in \operatorname{Ran} P_{ \pm}\left(J_{0} P_{\mathrm{ac}}(A) ; A\right)$ then

$$
\lim _{t \rightarrow \mp \infty}\left\|e^{-i t A} \phi-e^{-i t B} \eta_{ \pm}\right\|=0
$$

where $\eta_{ \pm}=\Omega^{ \pm}\left(B, A ; J_{0}\right) \phi$.

Proof. It follows from Proposition 2.2 that $\Omega^{ \pm}\left(B, A ; J_{0}\right) \phi=0$ if $\phi \perp \operatorname{Ran} P_{ \pm}\left(J_{0} P_{\mathrm{ac}} \times\right.$ $(A) ; A)$. On the other hand if $\phi \in \operatorname{Ran} P_{ \pm}\left(J_{0} P_{\mathrm{ac}}(A) ; A\right)$ then

$$
\left\|\Omega^{ \pm}\left(B, A ; J_{0}\right) \phi\right\|=\left\|s-\lim e^{i t B} e^{-i t A} \phi\right\|=\|\phi\| .
$$

We conclude that $\Omega^{ \pm}$are partial isometries with initial space $\operatorname{Ran} P_{ \pm}\left(J_{0} P_{\text {ac }}(A) ; A\right)$. Similarly $\left(\Omega^{ \pm}\right)^{*}$ has initial space $P_{ \pm}\left(J_{0}^{*} P_{\text {ac }}(B) ; B\right)$.

\section{§3. One Dimension}

Although our results in this section are mainly specialisations of those in Section 5, they are presented here because of their technical and conceptual simplicity. Throughout this section $H_{0}$ will denote the operator $-\frac{d^{2}}{d x^{2}}$ on $L^{2}(\mathbb{R})$. We let $p=-i \frac{d}{d x}$.

Definition. We say that the potential $V$ lies in $\mathscr{P}$ if and only if its positive part $V_{+}$lies in $L_{\text {loc }}^{1}$ and its negative part $V_{-}$obeys $Q\left(V_{-}\right) \subset Q\left(H_{0}\right)$ and

$$
\left(\phi, V_{-} \phi\right) \leqq \alpha\left(\phi, H_{0} \phi\right)+\beta(\phi, \phi)
$$

for some $\alpha<1$ and all $\phi \in Q\left(H_{0}\right)$. Given $V \in \mathscr{P}$ one can define the quadratic form sum $H=H_{0}+V$ with form domain $Q\left(H_{0}\right) \cap Q\left(V_{+}\right)$. We denote the set of such Hamiltonians $H$ by $\mathcal{O}$.

Since $Q\left(H_{0}\right) \supset Q(H)$ we have 
Lemma 3.1. Let $H \in \mathcal{O}$ and let $E<\inf \operatorname{spec}(H)$. Then $p(H-E)^{-1 / 2}$ is bounded with a bound on the norm which only depends on the $\alpha, \beta$ of Equation (3.1).

We shall need to use the space

$$
l_{1}\left(L^{2}\right)=\left\{f: \sum_{n=-\infty}^{\infty}\left(\int_{n}^{n+1}|f(x)|^{2} d x\right)^{1 / 2}<\infty\right.
$$

introduced by Birman and Solomjak [7]. Roughly speaking $l_{1}\left(L^{2}\right)$ requires $r^{-1-\varepsilon}$ fall-off at infinity. We note that if $f, g$ are non-zero then $f(x) g(p)$ is trace class if and only if both $f$ and $g$ lie in $l_{1}\left(L^{2}\right)$ (see e.g. [42]).

Theorem 3.2. If $f \in l_{1}\left(L^{2}\right)$ and $H \in \mathcal{O}$ then $f(x)(H-E)^{-1}$ is trace class for all $E \notin \operatorname{spec}(H)$.

Proof. We write $\|\cdot\|_{1}$ for the trace norm and assume, without loss, that $E<\inf$ $\operatorname{spec}(H)$. Suppose that we can show that

$$
\left\|f(x)(H-E)^{-1}\right\|_{1}<c\|f\|_{2}
$$

for all $f \in L^{2}(0,1)$, with $c$ depending only on the $\alpha, \beta$ of (3.1). Then by translation covariance (3.2) holds for $f \in L^{2}(-n, n)$ so that if $f \in l_{1}\left(L^{2}\right)$ and $\chi_{n}$ denotes the characteristic function of $(-n, n)$

$$
\begin{aligned}
\left\|f(H-E)^{-1}\right\|_{1} & \leqq \sum_{n=-\infty}^{\infty}\left\|f \chi_{n}(H-E)^{-1}\right\|_{1} \\
& \leqq \sum_{n=-\infty}^{\infty} c\left\|f \chi_{n}\right\|_{2}<\infty
\end{aligned}
$$

Pick $\eta \in C_{0}^{\infty}$ equal to one on $[0,1]$. Then

$$
\begin{aligned}
f(H-E)^{-1}= & f(H-E)^{-1} \eta+f\left[\eta,(H-E)^{-1}\right] \\
= & f(H-E)^{-1} \eta+f(H-E)^{-1}[H-E, \eta](H-E)^{-1} \\
= & f(H-E)^{-1} \eta+f(H-E)^{-1} p\left(-i \eta^{\prime}\right)(H-E)^{-1} \\
& +f(H-E)^{-1}\left(-i \eta^{\prime}\right) p(H-E)^{-1}
\end{aligned}
$$

given $f \in L^{2}(0,1)$. By Lemma 3.1 it suffices to show that $f(H-E)^{-1 / 2}$ and $(H-E)^{-1 / 2} \eta^{\prime}$ are Hilbert-Schmidt. But $\left(H_{0}-E\right)^{1 / 2}(H-E)^{-1 / 2}$ is bounded by Lemma 3.1 and $f\left(H_{0}-E\right)^{-1 / 2}$ and $\left(H_{0}-E\right)^{-1 / 2} \eta^{\prime}$ are easily seen to be HilbertSchmidt. It is straightforward to check that the norm estimate of (3.2) is satisfied.

Theorem 3.3. Let $H \in \mathcal{O}$. Then there are four projections $P_{l}^{ \pm}$and $P_{r}^{ \pm}$such that

(i) $P_{\mathrm{ac}}=P_{l}^{+}+P_{r}^{+}=P_{l}^{-}+P_{r}^{-}$

(ii) $\phi \in \operatorname{Ran} P_{l}^{ \pm}$if and only if

$$
\lim _{t \rightarrow \mp \infty} \int_{a}^{\infty}\left|\left(e^{-i t H} \phi\right)(x)\right|^{2} d x=0
$$

for all $a \in(-\infty, \infty)$, and similarly for $P_{r}^{ \pm}$with $\int_{a}^{\infty}$ replaced by $\int_{-\infty}^{a}$.

Proof. Let $j_{a}$ be a $C^{\infty}$ function which equals 1 for $x<(a-1)$ and 0 for $x>a$. 
since $j_{a}=j_{a}^{*}$ and $\left(j_{a}^{2}-j_{a}\right), j_{a}^{\prime}$ lie in $C_{0}^{\infty}$, we see by Lemma 3.1 and Theorem 3.2 that $\left(j_{a}^{2}-j_{a}\right)(H-E)^{-1}$ is compact and $(H-E)^{-1}\left[H, j_{a}\right](H-E)^{-1}$ is trace class. By Proposition 2.1, $P_{+}\left(j_{a} P_{\mathrm{ac}}(H) ; H\right)$ exists and since $\left(j_{a}-j_{b}\right)(H-E)^{-1}$ is compact it is independent of $a$; we call it $P_{l}^{ \pm}$or $P_{l}^{ \pm}(H)$ whenever we need to make explicit its dependence on $H$. We similarly write $P_{r}^{ \pm}$for $P_{ \pm}\left(\left(1-j_{a}\right) P_{\mathrm{ac}}(H) ; H\right)$ and deduce (i) from

$$
P_{ \pm}\left(j_{a} P_{\mathrm{ac}}(H) ; H\right)+P_{ \pm}\left(\left(1-j_{a}\right) P_{\mathrm{ac}}(H) ; H\right)=P_{ \pm}\left(P_{\mathrm{ac}}(H) ; H\right)=P_{\mathrm{ac}}(H) .
$$

The equivalence of $\phi \in \operatorname{Ran} P_{l}^{ \pm}$with (3.3) follows by Proposition 2.2, as does the corresponding result for $P_{r}^{ \pm}$.

Theorem 3.3 has a direct scattering interpretation. It says that any $\phi \in \operatorname{Ran} P_{\mathrm{ac}}$ is the sum of a wave which comes in from the left and one which comes in from the right as $t$ grows from $-\infty$. We explore this further in Section 4. For a conventional scattering theory we need a comparison dynamics.

Theorem 3.4. Let $H=H_{0}+V$ and $H_{l}=H_{0}+V_{l}$ lie in $\mathcal{O}$ and suppose that $\left(V-V_{l}\right) \chi_{(-\infty, a)}$ lies in $l_{1}\left(L^{2}\right)$ for some a, where $\chi_{(-\infty, a)}$ is the characteristic function of $(-\infty, a)$. Then

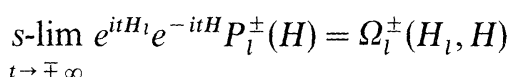

exists and defines a partial isometry from $\operatorname{Ran} P_{l}^{ \pm}(H)$ to $\operatorname{Ran} P_{l}^{ \pm}\left(H_{l}\right)$. In particular, for any $\phi \in \operatorname{Ran} P_{l}^{ \pm}(H)$ there exist $\eta_{ \pm}$such that

$$
\lim _{t \rightarrow \mp \infty}\left\|e^{-i t H_{l}} \eta_{ \pm}-e^{-i t H} \phi\right\|=0
$$

Proof. By Lemma 3.1 and Theorem 3.2, $\left(H_{l}-E\right)^{-1}\left(H_{l} j_{a}-j_{a} H\right)(H-E)^{-1}$ is trace

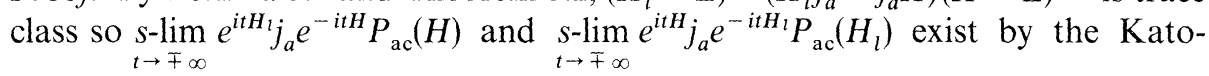
Birman theory $[33,36]$. Proposition 2.3 completes the proof.

Example 1. Let $V$ be continuous on $(-\infty, \infty)$ with

$$
\lim _{x \rightarrow \pm \infty}|x|^{1+\varepsilon}\left|V(x)-a_{ \pm}\right|=0
$$

with $a_{+} \neq a_{-}$allowed. If we put $H_{l}=H_{0}+a_{-}$and $H_{r}=H_{0}+a_{+}$we have a complete scattering theory with two "channels". This is somewhat special in that $P_{l}^{+}\left(H_{l}\right)=1-P_{r}^{+}\left(H_{r}\right)$, something which is definitely not required.

Example 2. Let $V$ be continuous on $(-\infty, \infty)$ with

$$
\lim _{x \rightarrow-\infty}|x|^{1+\varepsilon}|V(x)|=0
$$

and

$$
\lim _{x \rightarrow+\infty} V(x)=\infty .
$$

Take $H_{l}=H_{0}$ and $H_{r}=H_{0}+V(x)+V(-x)$. Then $H_{r}$ has purely discrete spectrum so that $P_{l, r}^{ \pm}\left(H_{r}\right)=0$. Hence $P_{r}^{ \pm}(H)=0$ so $P_{l}^{+}(H)=P_{l}^{-}(H)=P_{\mathrm{ac}}(H)$ and "everything bounces of the wall". 
Example 3. Let $W(x)$ be a periodic $L_{\text {loc }}^{1}$-function. Let $V$ be a function in $L_{\text {loc }}^{1}$ such that $\chi_{(-\infty, a)} V$ and $\chi_{(b, \infty)}(V-W)$ lie in $l^{1}\left(L^{2}\right)$ for some $a$ and $b$. Then one can take $H_{l}=H_{0}$ and $H_{r}=H_{0}+W$. In particular $H \mid \operatorname{Ran} P_{r}^{ \pm}(H)$ is unitarily equivalent to $H_{r} \mid \operatorname{Ran} P_{r}^{ \pm}\left(H_{r}\right)$. Clearly $\sigma\left(H_{r} \mid \operatorname{Ran} P_{r}^{ \pm}\left(H_{r}\right)\right) \subset \sigma\left(H_{r}\right)$; in fact we shall show in Section 4 that the spectra are equal in this case (but the multiplicity is 1 for $H_{r} \mid$ Ran $P_{r}^{ \pm}$and 2 for $H_{r}$ itself). Thus for any interval $I$ disjoint from $\sigma\left(H_{r}\right)$-a gap$E_{I}(H) P_{l}^{+}=E_{I}(H) P_{l}^{-}$and we have answered Papanicolaou's problem.

We shall show in Section 6 that the Hamiltonians of these three examples have no singular continuous spectrum if the potentials approach their asymptotic values exponentially. This condition is surely unnecessarily strong. We next note that

Proposition 3.5. $H \mid \operatorname{Ran} P_{l}^{+}$is antiunitarily equivalent to $H \mid \operatorname{Ran} P_{l}^{-}$under complex conjugation. Hence their spectral multiplicities are equal.

Proof. If $C \psi=\bar{\psi}$ then $C H C=H$ so

$$
C\left(e^{i t H} j_{a} e^{-i t H}\right) C=e^{-i t H} j_{a} e^{i t H}
$$

and thus $C P_{l}^{+}=P_{l}^{-}$. It follows that

$$
C\left(H P_{l}^{+}\right) C=H P_{l}^{-} \text {. }
$$

Finally we consider extensions of the theory to potentials which may diverge to $-\infty$ as $x \rightarrow \pm \infty$. For simplicity we suppose that $V \in L_{\text {loc }}^{2}$ with $\int_{-n}^{n}|V(x)|^{2} d x$ polynomially bounded as $n$ increases. We let $H$ denote some self-adjoint extension of $\left(H_{0}+V\right)$ defined on $\mathscr{D}_{0}=\left\{\eta \in L^{\infty} \cap \operatorname{Dom} H_{0}: \lim _{x \rightarrow \infty}(1+x)^{m} \sup _{|y|>x}|\eta(y)|=0\right.$ for all $\mathrm{m}\}$. The point is that if $\phi \in L^{2}$ has compact support then

$$
\begin{aligned}
& \left(H_{0}+1-i\right)^{-1} \phi \in \mathscr{D}_{0}, \text { so } \\
& \left(H_{0}+1-i\right)^{-1} j=\left(H_{0}+1-i\right)^{-1} j-(H+1-i)^{-1} V\left(H_{0}+1-i\right)^{-1} j
\end{aligned}
$$

for any bounded function $j$ of compact support (applied to all functions in $L^{2}$ ) and for any $L^{2}$ function $j$ of compact support (applied to all functions in $C_{0}^{\infty}$ ). The following is motivated by ideas from [6] and [10].

Theorem 3.6. Let $V, H$ be as above. If $j \in L^{2}$ has compact support then $(H+1-i)^{-1} j$ is trace class. If $\eta \in C_{0}^{\infty}$ then $(H+1-i)^{-1} \eta p$ is bounded.

Proof. Let $\phi \in C_{0}^{\infty}$ equal 1 on the support of $j$. Then

$$
(H+1-i)^{-1} V\left(H_{0}+1-i\right)^{-1} j=(H+1-i)^{-1} V \phi\left(H_{0}+1-i\right)^{-1} j+C
$$

where

$$
\begin{aligned}
C & =(H+1-i)^{-1} V\left(H_{0}+1-i\right)^{-1}\left[\phi, H_{0}\right]\left(H_{0}+1-i\right)^{-1} j \\
& =\left\{\left(H_{0}+1-i\right)^{-1}-(H+1-i)^{-1}\right\}\left[\phi, H_{0}\right]\left(H_{0}+1-i\right)^{-1} j
\end{aligned}
$$

by (3.4). Using (3.4) once more leads to

$$
(H+1-i)^{-1} j=A_{1}+A_{2}+A_{3}+A_{4}
$$


where

$$
\begin{aligned}
& A_{1}=\left(H_{0}+1-i\right)^{-1} j \\
& A_{2}=-(H+1-i)^{-1} V \phi\left(H_{0}+1-i\right)^{-1} j \\
& A_{3}=\left\{\left(H_{0}+1-i\right)^{-1}-(H+1-i)^{-1}\right\} \phi^{\prime \prime}\left(H_{0}+1-i\right)^{-1} j \\
& A_{4}=\left\{\left(H_{0}+1-i\right)^{-1}-(H+1-i)^{-1}\right\} 2 i \phi^{\prime} p\left(H_{0}+1-i\right)^{-1} j .
\end{aligned}
$$

By a Sobolev estimate $\left(H_{0}+1-i\right)^{-1 / 2} W$ is bounded if $W \in L^{2}$. This together with (3.5) implies the boundedness of $(H+1-i)^{-1} j$. Replacing $j$ by $\eta p$ in (3.5) where $\eta \in C_{0}^{\infty}$ and $\phi \in C_{0}^{\infty}$ equals 1 on the support of $\eta$ we similarly find that $(H+1-i)^{-1} \eta p$ is bounded. Since $\left(H_{0}+1-i\right)^{-1} j$ is trace class and $(H+1-i)^{-1} V \phi$ is bounded and $(H+1-i)^{-1} \phi^{\prime} p$ is bounded we finally deduce using (3.5) that $(H+1-i)^{-1} j$ is trace class.

We immediately conclude from Theorem 3.6 that

Theorem 3.7. Theorem 3.3 extends to self-adjoint extensions $H$ of $\left(H_{0}+V\right)$ provided that $\int_{-n}^{n}|V(x)|^{2} d x$ is polynomially bounded as $n$ increases.

Example 4. Suppose that $V \in L_{\text {loc }}^{2}$ with $V(x) \geqq-\alpha x^{2}-\beta$. Then $H$ is essentially self-adjoint on $C_{0}^{\infty}$ and one has the ordinary kind of $P_{l, r}^{ \pm}$.

Example 5. Suppose that $H$ is limit circle (see [35]) at both $+\infty$ and $-\infty$. Then one can choose boundary conditions so that waves reaching $+\infty$ are sent back in at $-\infty$. Because of this coupling it seems surprising at first that $P_{l, r}^{ \pm}$exist. The point is that $P_{\mathrm{ac}}=0$ !

Example 6. Suppose that $V \rightarrow 0$ at $-\infty$ like $|x|^{-1-\varepsilon}$ but that $H$ is limit circle at $+\infty$. Then $P_{l}^{ \pm} \neq 0$ but $P_{r}^{ \pm}=0$. The boundary conditions at $+\infty$ must involve total reflection.

\section{§4. Geometric Scattering and Homogeneous One-dimensional Systems}

We use Theorem 3.3 to see how much scattering theory can be developed without reference to a comparison dynamics. Thus, rather than thinking of scattering as a perturbative phenomenon we consider correlations between the dynamics, $e^{-i t H}$, and the configuration space geometry, $x \rightarrow \pm \infty$. The description of scattering in terms of correlations between spatial and temporal asymptotics is not new; among previous examples of that philosophy we mention:

(1) The Lax-Phillips theory of acoustical scattering [29], which is the example par excellence of geometric scattering theory. It has been extensively developed but does not seem to be applicable to Schrödinger operators with potentials of infinite range. However, Enss [21] has recently developed an approach to scattering which overlaps the Lax-Phillips approach in some methodological aspects and our approach on some points of philosophy.

(2) Certain $C^{*}$-algebraic approaches to long range scattering $[5,9,22,28]$ are partly geometric in nature. Those that deal with asymptotic momenta can be thought of as looking at the asymptotic form of $e^{i t H} X e^{-i t H}$. 
(3) Various authors (see $[4,45]$ ) beginning with Ruelle [38] have attempted to describe the orthogonal complement of the span of the eigenvalues of $H$ in purely geometric terms.

(4) There have been attempts at describing the usual time-dependent theory in more geometric terms $[18,47]$.

We should distinguish our attempt to describe scattering theory in geometric terms using primarily time-dependent methods (mainly the Kato-Birman theory) from recent papers $[15,20,43,44]$ using geometric methods to study the more usual spectral and scattering problems.

We suppose throughout this section that $H \mid \operatorname{Ran} P_{l}^{+}$has simple spectrum. By using Dirichlet decoupling [14] and the fact that second order operators on $(0, \infty)$ with a regular boundary condition at 0 always have simple spectrum [8], it should not be hard to prove this hypothesis for all $V \in \mathscr{P}$. The operator $P_{l}^{+} P_{l}^{-} P_{l}^{+}$is a non-negative self-adjoint contraction on $\operatorname{Ran} P_{l}^{+}$which commutes with $H$, so by the simplicity hypothesis there is a function $R$ on $\sigma\left(H \mid \operatorname{Ran} P_{l}^{+}\right)$ such that $0 \leqq R(E) \leqq 1$ and

$$
P_{l}^{+} P_{l}^{-} P_{l}^{+}=R(H) \mid \operatorname{Ran} P_{l}^{+} .
$$

Moreover $R$ is uniquely determined almost everywhere with respect to Lebesgue measure by this equation. We call it the reflection modulus. It is easy to show that

Proposition 4.1. In the short range case $R(E)=|r(\sqrt{E})|^{2}$ where $r$ is the usual reflection coefficient.

There is an asymmetry in the definition of $R$, since we could just as well have considered $\tilde{R}$, defined by

$$
P_{l}^{-} P_{l}^{+} P_{l}^{-}=\tilde{R}(H) \mid \operatorname{Ran} P_{l}^{-} .
$$

Proposition. 4.2. $R(E)=\tilde{R}(E)$ for almost every $E$ in

$$
\sigma\left(H \mid \operatorname{Ran} P_{l}^{+}\right)=\sigma\left(H \mid \operatorname{Ran} P_{l}^{-}\right) .
$$

Proof. By Proposition 3.5, $C P_{l}^{-} C=P_{l}^{+}$where $C$ is complex conjugation. Since $R$ is real and

$$
C R(H) P_{l}^{+} C=C P_{l}^{+} P_{l}^{-} P_{l}^{+} C=P_{l}^{-} P_{l}^{+} P_{l}^{-}=\tilde{R}(H) P_{l}^{-}
$$

we see that $R=\tilde{R}$.

There is a second proof of Proposition 4.2 which is important because it implies a left-right symmetry.

Theorem 4.3. Let $A$ be a self-adjoint operator and $P, Q$ two orthogonal projections commuting with $A$. Then there is a partial isometry $U$ from $\operatorname{Ker}(P Q)^{\perp}$ which commutes with $A$ and satisfies

$$
P Q P=U(Q P Q) U^{*} \text {. }
$$

Proof. (Motivated by [13]). If $U$ is defined by the polar decomposition $P Q=$ $U|P Q|$ then since $P Q$ commutes with $A$ so does $U$. Moreover $Q P=\left\{P Q \mid U^{*}\right.$ so

$$
P Q P=(P Q)(Q P)=U|P Q|^{2} U^{*}=U(Q P Q) U^{*} .
$$


Theorem 4.3 provides a second proof of Proposition 4.2 and also leads to the following result.

Proposition 4.4. Define the function $R_{r}$ on $\sigma\left(H \mid \operatorname{Ran} P_{r}^{-}\right)$by

$$
P_{r}^{+} P_{r}^{-} P_{r}^{+}=R_{r}(H) \mid \operatorname{Ran} P_{r}^{+}
$$

and extend $R$ and $R_{r}$ to all of $(-\infty, \infty)$ by setting them equal to 1 outside $\sigma\left(H \mid \operatorname{Ran} P_{l}^{+}\right)$, resp. $\sigma\left(H \mid \operatorname{Ran} P_{r}^{+}\right)$. Then $R=R_{r}$ almost everywhere.

Proof. The functions $1-R(E)$ and $1-R_{r}(E)$ on $(-\infty, \infty)$ are the spectral functions of

$$
1-\left\{\left(1-P_{l}^{-}\right)+P_{l}^{-} P_{l}^{+} P_{l}^{-}\right\}=P_{l}^{-} P_{r}^{+} P_{l}^{-}
$$

and

$$
1-\left\{\left(1-P_{r}^{+}\right)+P_{r}^{+} P_{r}^{-} P_{r}^{+}\right\}=P_{r}^{+} P_{l}^{-} P_{r}^{+}
$$

respectively. By Theorem 4.3 these are equal.

Examples 1 to 3 of the last section show that $\sigma\left(H \mid \operatorname{Ran} P_{l}^{+}\right)$and $\sigma\left(H \mid \operatorname{Ran} P_{r}^{+}\right)$ need not be equal, so that the extension procedure used in Proposition 4.4 is important.

Returning now to the study of comparison dynamics, we feel that if one wants them to have a reasonable physical interpretation the following restriction is necessary.

Definition. A Hamiltonian $H$ in $\mathcal{O}$ is called homogeneous if and only if

(i) $P_{l}^{+}=P_{r}^{-}$(no reflection)

(ii) $H$ has purely absolutely continuous spectrum.

These criteria are useful for understanding why the free field is a suitable comparison dynamics in the Haag-Ruelle theory [25, 36, 39] and for understanding the choice of comparison dynamics in magnon scattering $[23,36,46]$.

Example. $H_{0}=-\frac{d^{2}}{d x^{2}}$ is homogeneous since $P_{l}^{+}$and $P_{r}^{-}$both have range

$$
\{f: \hat{f}(k)=0 \text { for } k \leqq 0\}
$$

where denotes the Fourier transform.

Theorem 4.5. If $V$ is periodic and in $L_{\mathrm{loc}}^{1}$, then $H$ is homogeneous.

Proof. Without loss suppose that $V(x+1)=V(x)$. The analysis of $H$ as an explicit direct integral $[19,31,37]$ shows that (ii) is obeyed. Moreover there is an eigenfunction expansion

$$
\begin{aligned}
& \hat{f}_{n}(k)=\int_{-\infty}^{\infty} \overline{\phi_{n}(x, k)} f(x) d x \quad k \in[-\pi, \pi] \\
& f(x)=\sum_{n=1}^{\infty} \int_{-\pi}^{\pi} \phi_{n}(x, k) \hat{f}_{n}(k) d k \\
& \widehat{H f}_{n}(k)=\varepsilon_{n}(k) \hat{f}_{n}(k)
\end{aligned}
$$


where

(a) $\varepsilon_{n}(k)$ is real analytic and non-constant on $(-\pi, \pi)$.

(b) $u_{n}(x, k) \equiv e^{-i k x} \phi_{n}(x, k)$ is a periodic function of $x$ with period 1 and if $x$ is restricted to $(0,1)$ is an analytic function of $k$ on $(-\pi, \pi)$ with values in $L^{2}(0,1)$.

Homogeneity is proven if we show that $\operatorname{Ran} P_{l}^{+}$and $\operatorname{Ran} P_{r}^{-}$are both equal to

$$
\left\{f: \hat{f}_{n}(k)=0 \text { for those } k, n \text { with } \frac{\partial \varepsilon_{n}(k)}{\partial k} \leqq 0\right\} \text {. }
$$

This follows by a density argument if we show that if $[a, b]$ is an interval on which $\frac{\partial \varepsilon_{n}(k)}{\partial k}>0($ resp. $<0)$ and $g \in C_{0}^{\infty}(a, b)$ then

$$
f(x) \equiv \int_{a}^{b} \phi_{n}(x, k) g(k) d k
$$

obeys

$$
\int_{0}^{1}\left|\left(e^{-i t H} f\right)(x+y)\right|^{2} d y \leqq C_{N}(1+|x|+|t|)^{-N}
$$

provided $x / t \leqq 0($ resp. $x / t \geqq 0)$. (4.1) is proven by a stationary phase argument $[24,36]$. Starting from

$$
\begin{aligned}
& \left(e^{-i t H} f\right)(x)=\int_{a}^{b} u_{n}(x, k) g(k) e^{-i\left(t \varepsilon_{n}(k)-x k\right)} d k \\
& =\int_{a}^{b} u_{n}(x, k) g(k)\left[i\left(t \frac{\partial \varepsilon_{n}}{\partial k}-x\right)^{-1} \frac{\partial}{\partial k}\right]^{N} e^{-i\left(t \varepsilon_{n}(k)-x k\right)} d k
\end{aligned}
$$

we integrate by parts $N$ times and use the fact that, since $u$ is periodic,

$$
\int_{0}^{1}\left|\frac{\partial^{l} u}{\partial k^{l}}(x+y)\right|^{2} d y
$$

is uniformly bounded in $x$, to deduce that the left-hand side of (4.1) is dominated by

$$
C_{N} \sup _{a<k<b}\left|t \frac{\partial \varepsilon_{n}}{\partial k}-x\right|^{-N} \text {. }
$$

This yields (4.1) since $\frac{\partial \varepsilon_{n}}{\partial k}$ has a positive lower bound on $[a, b]$.

We note the following restatement of a theorem of Levinson-see Deift and Trubowitz [16] for extensive discussion and proofs.

Theorem 4.6. Let $V$ be short range in a suitable sense, for example

$$
\int_{-\infty}^{\infty}\left(1+x^{2}\right)|V(x)|^{2} d x<\infty
$$

suffices, and let $H=H_{0}+V$ be homogeneous. Then $V$ is zero almost everywhere. 
Consideration of this example demonstrates the importance of condition (ii) in the definition of homogeneity. There are reflectionless short range potentials ("KdV solitons" see e.g. $[13,16]$ ) that have non-empty point spectrum. We feel that the theory of homogeneous Hamiltonians is worthy of further study, and pose the following unsolved problems.

Question 1. If $V$ is almost periodic is $H_{0}+V$ homogeneous? It may be possible to answer this for a large class of almost periodic $V$ using the results of Dinaburg and Sinai [17].

Question 2. Can one find a proof that $H_{0}$ is homogeneous without using the Fourier transform? This might yield a simplification in the proof of Theorem 4.5 and even a solution of Question 1.

Question 3. Let $W$ be periodic and $V$ short range. If $H_{0}+W+V$ is homogeneous, does it follow that $V$ is zero almost everywhere?

Question 4. Are there any homogeneous Hamiltonians $H \in \mathcal{O}$ for which $V$ is not almost periodic?

\section{§5. Systems Periodic in All but One Dimension}

In this section we consider potentials $V \in \mathscr{P}$ on $\mathbb{R}^{v}$ for which there exist $v-1$ periods, that is linearly independent vectors $a_{1}, \ldots, a_{v-1}$ such that $V\left(x+a_{j}\right)=V(x)$. Without loss of generality we can suppose that the span of $a_{1}, \ldots, a_{v-1}$ is $\{(0$, $\left.\left.x_{2}, \ldots, x_{v}\right)\right\}$.

Example 1 (Infinite Sheet). Let $W$ be a potential on $\mathbb{R}^{v}$ such that

$$
|W(x)| \leqq C(1+|x|)^{-v-\varepsilon} .
$$

Let $L$ be the lattice of integral combinations of $a_{1}, \ldots, a_{v-1}$ and define

$$
V(x)=\sum_{b \in L} W(x+b) .
$$

Then $-\Delta+V$ describes scattering from an "infinite sheet" or "thin film" of scatterers, each of which is described by a potential $W$ centred at a point of the lattice. Notice that

$$
|V(x)| \leqq C\left(1+\left|x_{1}\right|\right)^{-1-\varepsilon} .
$$

For this example we recover the result of [11] that $\Omega^{ \pm}(-\Delta+V,-\Delta)$ exist and are complete in the sense that their ranges are equal. It may happen that this common range is not all of $\operatorname{Ran} P_{\mathrm{ac}}(-\Delta+V)$ as we shall see.

Example 2 (Half-solid). Let $W$ be a function on $\mathbb{R}^{v}$ which has $v$ periods and suppose that the plane $x_{1}=0$ has rational indices, that is there is a set of $v-1$ independent integral combinations of the periods which lie in the plane. Let $V(x)=0$ if $x_{1}<0$ and $V(x)=W(x)$ if $x_{1} \geqq 0$. Then $-\Delta+V$ is a direct analogue of the Papanicolaou example. Our analysis below implies the existence of plane scattering waves from the left and Bloch scattering waves from the right. We 
have completeness in the sense that the span of the two types of wave from the past equals their span from the future, and in particular there is complete reflection from the interface at energies in "gaps" for the solid.

Example 3 (Lattice Dislocations). Let $W_{1}$ and $W_{2}$ be two potentials each periodic with $v$ periods, which may be distinct for the two potentials, and suppose that the plane $x_{1}=0$ contains a set of $v-1$ independent vectors which are integral combinations of each of the two sets of periods. Let $V(x)=W_{1}(x)$ if $x_{1}<0$ and $V(x)=W_{2}(x)$ if $x_{1} \geqq 0$. Our results below yield existence and completeness in the same sense as in Example 1. The case of particular physical interest is where $W_{2}(x)=W_{1}(T x)$ with $T$ a Euclidean transformation. The plane $x_{1}=0$ is then a "lattice dislocation", called a shear if $T$ is purely translational.

The restriction to rational indices in Examples 2 and 3 is an annoying one from a mathematical point of view, and arises from the fact that for a direct integral decomposition of the type we shall use, strict periodicity is essential. This emphasises the mathematical need for methods of analysis applicable to almost periodic Schrödinger operators. However, from a physical point of view the restriction to rational indices is not so serious, for as J. Hopfield has emphasised to us, a microscopic view of sliding together with the notion that an atom cannot be sliced in half indicates that almost periodicity is impossible for the kind of physical systems we are considering.

Motivated by [11] we now proceed to make the direct integral decomposition of $L^{2}\left(\mathbb{R}^{v}\right)$ induced by the group of symmetries $x \rightarrow x+\sum_{i=1}^{v-1} n_{i} a_{i}$. Let $C$ be the cylinder over a basic cell for the periods, so that $x \in C$ if and only if

$$
\left(0, x_{2}, \ldots, x_{v}\right)=\sum_{i=1}^{v-1} y_{i} a_{i}
$$

where $0 \leqq y_{i}<1$. Let $\theta \in T \equiv[0,2 \pi)^{v-1}$ and let $H_{0}(\theta)$ be $-\Delta$ on $L^{2}\left(C, d^{v} x\right)$ with the domain given by requiring that the set of $f \in L^{2}(C)$ which have $C^{\infty}$ extensions to $\mathbb{R}^{v}$ such that

$$
f\left(x+a_{j}\right)=e^{i \theta_{j}} f(x)
$$

is a core for $H(\theta)$. Finally let $H(\theta)=H_{0}(\theta)+V$. Then by $[19,37]$

$$
H \equiv-\Delta+V \simeq \int_{T}^{\oplus} H(\theta) d \theta
$$

and for any function $f$ on $\mathbb{R}$ multiplication by $f\left(x_{1}\right)$ in $L^{2}\left(\mathbb{R}^{v}\right)$ is just $\int_{T}^{\oplus} f_{\theta} d \theta$, where $f_{\theta}$ is multiplication by $f\left(x_{1}\right)$ in the fibre $L^{2}(C)$ corresponding to $\theta^{T}$; that is the decomposition respects geometry in the $x_{1}$-direction.

We begin by analysing $H(\theta)$ by a method analogous to that in Section 3 . Before starting we note that if $\mathscr{P}, \mathcal{O}$ are defined as in Section 3 and $V \in \mathscr{P}$ then (3.1) holds for all $\theta$, with $H_{0}$ replaced by $H_{0}(\theta)$. We shall need to use the space

$$
l_{1}\left(L^{p}(C)\right) \equiv\left\{g: \sum_{n=-\infty}^{\infty}\left(\int_{\substack{x \in C \\ n \leqq x_{1}<n+1}}|g(x)|^{p} d^{v} x\right)^{1 / p}<\infty\right\} .
$$


Theorem 5.1. Let $V \in \mathscr{P}$ and $f \in l_{1}\left(L^{q}(C)\right)$ for some $q \geqq 2, q>v$. Then if $E \notin \sigma(H(\theta))$

(a) $\partial_{1}(H(\theta)-E)^{-1 / 2}$ is bounded

(b) $f(H(\theta)-E)^{-k}$ is trace class for sufficiently large $k$.

Proof. (a) This is identical to the proof of Lemma 3.1.

(b) This is similar to but somewhat more complicated than Theorem 3.2. Namely as in that case we can suppose that $f(x)$ is zero unless $x_{1} \in[0,1]$, so that $f \in L^{q}$. Let $\eta \in C_{0}^{\infty}(\mathbb{R})$ equal one for $x_{1} \in[0,1]$ and regard $\eta$ as a function on $C$. If $p=-i \partial_{1}$ then

$$
\begin{aligned}
f(H-E)^{-n-1}= & f(H-E)^{-1} \eta(H-E)^{-n}+2 f(H-E)^{-1} p\left(-i \eta^{\prime}\right)(H-E)^{-n-1} \\
& +f(H-E)^{-1} \eta^{\prime \prime}(H-E)^{-n-1}
\end{aligned}
$$

where we do not make explicit the dependence of $H(\theta)$ on $\theta$. We claim that $f(H-E)^{-k}$ lies in the trace ideal $\mathscr{I}_{a(k)}$ where $a(k)=\max (1, q / k)$. This is proven inductively, the result holding for $k=1$ by the following lemma. But part (a), the lemma below, and (5.1) together show by Hölder's inequality that $f(H-E)^{-k} \in \mathscr{I}_{a(k)}$ implies $f(H-E)^{-k-1} \in \mathscr{I}_{a(k+1)}$.

Lemma 5.2. If $q \geqq 2, q>v$ and $f \in L^{q}(C)$

then $f(H(\theta)-E)^{-1 / 2} \in \mathscr{I}_{q}$.

Proof. As $\left(H_{0}(\theta)-E\right)^{1 / 2}(H(\theta)-E)^{-1 / 2}$ is bounded we need only prove that $X \equiv f\left(H_{0}(\theta)-E\right)^{-1 / 2} \in \mathscr{I}_{q}$. This is done by Fourier analysis, considering $C$ as a locally compact abelian group with dual group $\hat{C}$. Regarded as an operator on $L^{2}(\hat{C})$ one sees that $\left(H_{0}(\theta)-E\right)^{-1 / 2}$ is multiplication by a function $g$ which lies in $L^{q}(\hat{C})$ for all $q>v$. Hence $X=f(Q) g(P)$ lies in $\mathscr{I}_{q}$ by complex interpolation between $q=2$ and $q=\infty($ see $[42])$.

Theorem 5.3. If $H \in \mathcal{O}$ then

$$
P_{\mathrm{ac}}(\theta)=P_{l}^{+}(\theta)+P_{r}^{+}(\theta)=P_{l}^{-}(\theta)+P_{r}^{-}(\theta)
$$

for all $\theta$. Moreover $\eta \in \operatorname{Ran} P_{l}^{ \pm}(\theta)$ if and only if $\eta \in \operatorname{Ran} P_{\mathrm{ac}}(\theta)$ and

$$
\lim _{t \rightarrow \pm \infty} \int_{x_{1} \geqq a}\left|e^{-i t H(\theta)} \eta(x)\right|^{2} d^{v} x=0
$$

for all $a$.

Proof. By Proposition 2.1 it suffices to show that $j_{a} Q(H(\theta)) \subset Q(H(\theta))$ and that

$$
E_{I}(H(\theta))\left[j_{a}, H(\theta)\right] E_{I}(H(\theta)) \in \mathscr{I}_{1}
$$

for all $I, \theta$, where $j_{a}$ is a $C^{\infty}$ function of $x_{1}$ which equals 0 (resp. 1) if $x<a-1$ (resp. $x>a+1$ ) and $E_{I}$ is the spectral projection of the bounded interval $I$. The first condition is implied by the trivial inclusions $j_{a} Q\left(H_{0}(\theta)\right) \subset Q\left(H_{0}(\theta)\right)$ and $j_{a} Q\left(V_{+}\right) \subset Q\left(V_{+}\right)$. The second condition is a consequence of the identity

$$
E_{I}\left[j_{a}, H(\theta)\right] E_{I}=E_{I}\left(\partial_{1} j_{a}-j_{a} \partial_{1}\right) E_{I}
$$

and the results of Theorem 5.1.

In Appendix 2 we shall prove that $P_{\mathrm{pp}}(\theta)$ is measurable in $\theta$. Since $P_{\text {sing }}(\theta)=0$ 
for all $\theta$ by Appendix 1 it follows that $P_{\mathrm{ac}}(\theta)$ and hence

$$
P_{l}^{+}(\theta)=\underset{t \rightarrow-\infty}{s-\lim } e^{i t H(\theta)} j_{a} e^{-i t H(\theta)} P_{\mathrm{ac}(\theta)}
$$

are measurable. There is therefore no difficulty in defining projections such as

$$
\tilde{P}_{\mathrm{ac}}=\int_{T}^{\oplus} P_{\mathrm{ac}}(\theta) d \theta, \quad P_{l, r}^{ \pm}=\int_{T}^{\oplus} P_{l, r}^{ \pm}(\theta) d \theta .
$$

Proposition 5.4.

$$
\begin{gathered}
\tilde{P}_{\mathrm{ac}}=P_{l}^{+}+P_{r}^{+}=P_{l}^{-}+P_{r}^{-} . \\
\text {If } \eta_{ \pm} \in \operatorname{Ran} P_{l}^{ \pm} \text {then } \\
\lim _{t \rightarrow \mp \infty} \int_{x_{1}>b}\left|\left(e^{-i t H} \eta_{ \pm}\right)(x)\right|^{2} d^{v} x=0
\end{gathered}
$$

for all $b$.

It is important to note that $\widetilde{P}_{\text {ac }}$ and $P_{\text {ac }}$ need not be equal. Each $H(\theta)$ might have an eigenvalue $\lambda(\theta)$ which is an analytic and non-constant function of $\theta$. Then by [37] any direct integral of the corresponding eigenvectors lies in

$$
\operatorname{Ran} P_{\mathrm{ac}} \cap\left(\operatorname{Ran} \tilde{P}_{\mathrm{ac}}\right)^{\perp} \text {. }
$$

It seems physically plausible that all $\eta$ in this subspace should be "surface states", that is should be "concentrated" near the surface for all time:

$$
\lim _{a \rightarrow \infty} \sup _{t} \int_{\left|x_{1}\right|>a}\left|\left(e^{-i t H} \eta\right)(x)\right|^{2} d^{v} x=0 .
$$

This would lead to a converse of Proposition 5.4.

It turns out that the truth of this conjecture is related to the question of whether the singular continuous spectrum $\sigma_{\text {sing }}(H(\theta))$ is empty. This is the problem we examine in the next section.

\section{§6. Surface States and Surface Impurities}

We continue with the notation of the last section. Since the projection $P_{\mathrm{pp}}(\theta)$ onto the span of the eigenvectors of $H(\theta)$ is measurable by Appendix 2, we can define the projection $P_{s}$ onto the "surface states" by

$$
P_{s}=\int_{T}^{\oplus} P_{\mathrm{pp}}(\theta) d \theta
$$

Proposition 6.1. If $\eta \in \operatorname{Ran} P_{s}$ then $\eta$ is "concentrated" near the surface $x_{1}=0$ for all time in the sense that

$$
\lim _{a \rightarrow \infty} \sup _{t} \int_{\left|x_{1}\right|>a}\left|\left(e^{-i t H} \eta\right)(x)\right|^{2} d^{v} x=0 .
$$

Proof. Since the direct integral decomposition respects respects the $x_{1}$-geometry

$$
\sup _{t} \int_{\left|x_{1}\right|>a}\left|\left(e^{-i t H} \eta\right)(x)\right|^{2} d^{v} x \leqq \int_{T} G(\theta, a) d \theta
$$


where

$$
G(\theta, a)=\sup _{t} \int_{\left|x_{1}\right|>a}\left|e^{-i t H(\theta)} \eta(\theta)\right|^{2} d^{v} x .
$$

Since $G(\theta, a) \leqq\|\eta(\theta)\|^{2} \in L^{1}(d \theta)$, (6.1) follows by the dominated convergence theorem if we show that

$$
\lim _{a \rightarrow \infty} G(\theta, a)=0
$$

for each $\theta$. By hypothesis $\eta(\theta)=\sum_{\alpha} C_{\alpha} \psi_{\alpha}$

where $\psi_{\alpha}$ are normalised eigenvectors of $H(\theta)$ and $\sum_{\alpha}\left|C_{\alpha}\right|^{2}=\|\eta(\theta)\|^{2}$. By a limiting argument we can suppose the sum is finite. In that case

$$
G(\theta, a) \leqq \sum_{\alpha, \beta}\left|C_{\alpha} C_{\beta}\right| \int_{\left|x_{1}\right|>a}\left|\psi_{\alpha}(x) \psi_{\beta}(x)\right| d^{v} x
$$

which goes to zero since $\psi_{\alpha} \psi_{\beta} \in L^{1}(C)$.

Corollary 6.2. Suppose that $H \in \mathcal{O}$ and that $\sigma_{\text {sing }}(H(\theta))=\varnothing$ for each $\theta$. Then $\eta \in \operatorname{Ran} P_{l}^{ \pm}$if and only if (5.2) holds for all $b$.

Proof. If $\eta \in \operatorname{Ran}\left(P_{r}^{+}+P_{s}\right)$ then by Propositions 5.4 and 6.1

$$
\lim _{a \rightarrow \infty} \lim _{t \rightarrow-\infty} \int_{x_{1} \leqq-a}\left|\left(e^{-i t H} \eta\right)(x)\right|^{2} d^{v} x=0 .
$$

The hypothesis on $\sigma_{\text {sing }}$ and Theorem 5.3 imply

$$
P_{l}^{+}(\theta)+P_{r}^{+}(\theta)+P_{\mathrm{pp}}(\theta)=1
$$

so by Proposition 5.4 we deduce that

$$
\lim _{a \rightarrow \infty} \lim _{t \rightarrow-\infty} \int_{x_{1} \leqq-a}\left|\left(e^{-i t H} \eta\right)(x)\right|^{2} d^{v} x=\left\|P_{l}^{+} \eta\right\|^{2}
$$

for all $\eta$. Thus (5.2) implies that $\|\eta\|^{2}=\left\|P_{l}^{+} \eta\right\|^{2}$ so that $\eta \in \operatorname{Ran} P_{l}^{+}$.

Therefore when $\sigma_{\text {sing }}(H(\theta))=\varnothing$ we have three scattering channels $P_{\mathrm{ac}, s}=$ $P_{\mathrm{ac}} P_{s}, P_{l}^{ \pm}, P_{r}^{ \pm}$with geometric interpretations for all three channels. There is however no scattering between the surface channel and the $l, r$ channels unless one introduces a localised "surface" impurity.

Theorem 6.3. Let $H=H_{0}+V$ where $V \in \mathscr{P}$ has $v-1$ periods and $\sigma_{\text {sing }}(H(\theta))=\varnothing$ for all $\theta$. Let $H^{\prime}=H_{0}+V+W$ where $W \in l_{1}\left(L^{q}\left(\mathbb{R}^{v}\right)\right)$ for some $q \geqq 2, q>v$. Then

$$
P_{\mathrm{ac}}\left(H^{\prime}\right)=P_{l}^{ \pm}\left(H^{\prime}\right)+P_{r}^{ \pm}\left(H^{\prime}\right)+P_{s}^{ \pm}\left(H^{\prime}\right)
$$

where $\eta \in \operatorname{Ran} P_{y}^{ \pm}\left(H^{\prime}\right)$ if and only if

$$
\lim _{a \rightarrow \infty} \lim _{t \rightarrow \mp \infty} \int_{x_{1} \in R_{y}(a)}\left|\left(e^{-i t I I} \eta\right)(x)\right|^{2} d^{v} x=0
$$

and

$$
R_{y}(a)= \begin{cases}\left\{x:\left|x_{1}\right|>a\right\} & \text { if } y=a c, s \\ \left\{x: x_{1}>-a\right\} & \text { if } y=l \\ \left\{x: x_{1}<a\right\} & \text { if } y=r .\end{cases}
$$


Proof. Combining the estimates given in the proofs of Theorems 5.1 and 5.3 with the Kato-Birman theory $[33,36]$ one sees that $\Omega^{ \pm}\left(H^{\prime}, H\right)$ exist and are complete. If $P_{y}^{ \pm}\left(H^{\prime}\right)$ is the projection with range

$$
\Omega^{ \pm}\left(H^{\prime}, H\right) P_{y}^{ \pm}(H) L^{2}\left(\mathbb{R}^{v}\right)
$$

then completeness and $\sum_{y} P_{y}^{ \pm}(H)=P_{\mathrm{ac}}(H)$ imply $\sum_{y} P_{y}^{ \pm}\left(H^{\prime}\right)=P_{\mathrm{ac}}\left(H^{\prime}\right)$. Now (6.3) holds for the Hamiltonian $H$ by Proposition 6.1 and Corollary 6.2, so it also holds for $H^{\prime}$ since $\eta \in \operatorname{Ran} P_{y}^{ \pm}\left(H^{\prime}\right)$ if and only if there exist $\phi \in P_{y}^{ \pm}(H)$ with

$$
\lim _{t \rightarrow \mp \infty}\left\|e^{-i t H^{\prime}} \eta-e^{i t H} \phi\right\|=0 \text {. }
$$

Thus scattering for the Hamiltonian $H^{\prime}$ may still be described in geometric terms, but there is now no reason why $P_{l, r} P_{s, \text { ac }}$ should vanish. Physically the impurity allows coupling between the surface and the $l, r$ channels.

This leaves the technical question of showing that $\sigma_{\text {sing }}(H(\theta))=\varnothing$. We shall prove this for the examples of Section 5, and most of those of Section 3. In all cases there are two potentials $W_{l}$ and $W_{r}$ such that

(i) If $W=W_{l} \chi_{(-\infty, 0)}+W_{r} \chi_{(0, \infty)}$ then $G=V-W$ converges to zero at $\pm^{\infty}$.

(ii) The spectral properties of $-\Delta+W_{l, r}$ are capable of detailed analysis.

In the rest of the section we shall suppose that $G$ lies in the space $L_{0}^{\infty}$ of bounded functions of compact support. One can easily accommodate local singularities of $G$ and exponential fall-off at infinity. With more work one could presumably develop the Agmon-Kuroda theory $[2,27,37]$ to allow $G$ which decay like $r^{-1-\varepsilon}$ at infinity. Since the cases of greatest interest have $G \in L_{0}^{\infty}$ we exploit the simplification then possible.

We shall reduce the analysis to a perturbative version of the limiting absorption method. We begin with an abstraction of this method motivated in part by the treatment in [36].

Definition. Let $\mathscr{H}=\bigoplus_{i=1}^{n} L^{2}\left(\mathbb{R}^{v}\right)$. Let $(a, b) \subset \mathbb{R}$ and let $Q \leqq \mathbb{R}^{v}$ an open set. We say that a self-adjoint operator $\mathrm{A}$ on lies in $\mathscr{A}(a, b, Q)$ if

(i) There is a complex neighbourhood $N$ of $(a, b)$ such that $f(A-z)^{-1} g$ has an analytic continuation from $N \cap\{\operatorname{Im} z>0\}$ to $N$ for all $f, g \in \bigoplus_{i=1}^{n} L_{0}^{\infty}\left(\mathbb{R}^{v}\right)$.

(ii) If $p=-i \partial$ and supp $f \subset Q$ then the continuation requirement is valid for $f \cdot p(A-z)^{-1} g$.

(iii) If $\operatorname{Im} z>0$ and $f \in \bigoplus_{i=1}^{n} L_{0}^{\infty}$ then $f(A-z)^{-1}$ is compact. If also supp $f \subset Q$ then $f \cdot p(A-z)^{-1}$ is compact.

Note. The case $Q \neq \mathbb{R}^{v}$ is only needed for some applications in the appendix.

A fundamental criterion for the absence of singular continuous spectrum in [37] immediately implies

Proposition 6.4. If $A \in \mathscr{A}(a, b, Q)$ then $\sigma_{\text {sing }}(A) \cap(a, b)=\varnothing$ and $\sigma_{\mathrm{pp}}(A)$ has no points of accumulation in $(a, b)$. 
The definition was chosen in order to make possible the following perturbation result.

Proposition 6.5. Let $f$ be an $n \times n$ matrix-valued function in $C_{0}^{\infty}$ with support in $Q$ and let $g$ be an $n \times n$ matrix-valued function in $L_{0}^{\infty}$ such that $(f \cdot p+g)$ is symmetric. If $A \in \mathscr{A}(a, b, Q)$ on $\bigoplus_{i=1}^{n} L^{2}\left(\mathbb{R}^{v}\right)$ then there exists a countable set $S \subset(a, b)$ with no limit points in $(a, b)$ such that if $(c, d) \subset(a, b) \backslash S$ then $(A+f \cdot p+g) \in \mathscr{A}(c, d, Q)$.

Proof. Let $B=f \cdot p+g$ and choose $\eta \in C_{0}^{\infty}$ equal to 1 on supp $f \cup \operatorname{supp} g$. We first claim that

$$
(A+B-z)^{-1} \eta=(A-z)^{-1} \eta\left(1+B(A-z)^{-1} \eta\right)^{-1}
$$

if $\operatorname{Im} z>0$ and the first inverse exists. This follows for large $\operatorname{Im} z$ by expanding the last inverse as a geometric series and using $\eta B=B$. If $f_{1}, g_{1} \in L_{0}^{\infty}$ and $\eta$ also equals 1 on supp $g_{1}$, it follows that

$$
f_{1}(A+B-z)^{-1} g_{1}=f_{1}(A-z)^{-1} \eta\left(1+B(A-z)^{-1} \eta\right)^{-1} g_{1} .
$$

By hypothesis and the analytic Fredholm theorem this can be continued from $N \cap\{\operatorname{Im} z>0\}$ to all of $N$ except for a set $P$ of poles with no limit points in $N$. Putting $S=P \cap(a, b)$ we obtain (i) for $A+B$, except that $S$ as constructed may depend on $g_{1}$ since we required $\eta \equiv 1$ on $\operatorname{supp} g_{1}$. But $S$ is independent of $f_{1}$ so by symmetry it is also independent of $g_{1}$. The proofs of (ii) and (iii) are similar.

Theorem 6.6. (a) If $A_{1}, \ldots, A_{n}$ lie in $\mathscr{A}(a, b, Q)$ for $L^{2}\left(\mathbb{R}^{v}\right)$ then $A_{1} \oplus \ldots \oplus A_{n}$ lies in $\mathscr{A}(a, b, Q)$ for $\bigoplus_{i=1}^{n} L^{2}\left(\mathbb{R}^{v}\right)$.

(b) $A=-\Delta$ lies in $\mathscr{A}\left(a, b, \mathbb{R}^{v}\right)$ for any $a, b>0$.

(c) Let $V \in L_{\mathrm{loc}}^{1}$ be periodic on $\mathbb{R}$. Then there is a countable set $E=\left\{x_{1}, x_{2}, \ldots\right\}$ such that $x_{n} \rightarrow \infty$ and $-\Delta+V \in \mathscr{A}(a, b, \mathbb{R})$ for all $(a, b)$ disjoint from $E$.

(d) Let $W$ be periodic on $\mathbb{R}^{v}$ with $(v-1)$ periods in $\left\{x_{1}=0\right\}$. Define $\mathscr{A}(a, b, Q)$

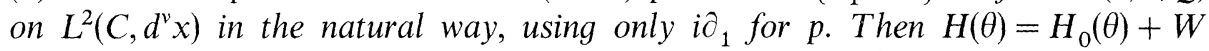
lies in $\mathscr{A}(a, b, C)$ for all $(a, b)$ disjoint from a set $E(\theta)=\left\{x_{1}(\theta), x_{2}(\theta), \ldots\right\}$ where $x_{n}(\theta) \rightarrow \infty$ as $n \rightarrow \infty$.

Proof. (a) is trivial.

(b) If $f, g \in L_{0}^{\infty}$ then for any $\phi \in L^{2}$ the Payley-Wiener theorem implies that $\widehat{f \phi}$ and $\widehat{g \phi}$ are entire functions with

$$
\sup _{|\operatorname{Im} k| \leqq \alpha}|\widehat{f \phi}(k)| \leqq C_{f, \alpha}\|\phi\|_{2}
$$

and a similar result with $f$ replaced by $g$. If $\operatorname{Im} z>0$ then by straightforward Fourier analysis

$$
\left(\phi, f(-\Delta-z)^{-1} g \psi\right)=\int_{C} \frac{\overline{f \phi(\bar{k}}) \widehat{g \psi}(k)}{k^{2}-z} d^{v} k
$$

where $C=\mathbb{R}^{v}$. As $z \rightarrow(a, b) \notin 0$ we can deform the contour to avoid singularities of $\left(k^{2}-z\right)^{-1}$ and use (6.5) to show that the analytic continuation is a bounded operator analytic in $z$. 
(c) This is similar to (b) once we note the existence of a Paley-Wiener type of theorem for the eigenfunction transform. In the notation of Theorem 4.5, if $f \phi \in L_{0}^{\infty} \times L^{2}$ then $f \phi_{n}$ can be analytically continued into a neighbourhood of $[-\pi, \pi]$. Thus

$$
\left(f \phi,(-\Delta+V-z)^{-1} g \psi\right)=\sum_{n} \int_{-\pi}^{\pi} \frac{\widehat{\widehat{f \phi}}_{n}(k) \widehat{g \psi}_{n}(k)}{\varepsilon_{n}(k)-z} d k
$$

can be continued past $(a, b)$ as long as $z$ avoids the discrete set

$$
\left\{\varepsilon_{n}(k): \frac{\partial \varepsilon_{n}}{\partial k}=0\right\} \cup\left\{\varepsilon_{n}( \pm \pi)\right\}
$$

(at the former points the contour may be pinched by two singularities).

(d) This is identical to (c); now $\varepsilon_{n}(k, \theta)$ depends on $\theta$ but since $\varepsilon_{n}(k, \theta) \rightarrow \infty$ as $n \rightarrow \infty$ for $\theta$ fixed, the proof goes through.

We can now prove

Theorem 6.7. Let $a_{1}, \ldots, a_{v-1}$ be $v-1$ periods in $\left\{x_{1}=0\right\}$ and let $a_{v}, b_{v}$ be two vectors each independent of $a_{1}, \ldots, a_{v-1}$. Let $W_{1}$ be bounded and periodic with periods $a_{1}, \ldots, a_{v}$ and $W_{2}$ bounded and periodic with periods $a_{1}, \ldots, a_{v-1}, b_{v}$. Suppose that $\mathrm{H}=\mathrm{H}_{0}+\mathrm{V}$ where

$$
V-W_{1} \chi_{(0, \infty)}-W_{2} \chi_{(-\infty, 0)} \in L_{0}^{\infty} .
$$

Then $\sigma_{\text {sing }}(H(\theta))=\varnothing$ for all $\theta$.

Proof. It is clearly sufficient to show that if

$$
\tilde{V}=W_{2} \chi_{(0, \infty)}+W_{1} \chi_{(-\infty, 0)}
$$

then

$$
A(\theta)=\left[\begin{array}{cc}
-\Delta+V & 0 \\
0 & -\Delta+\tilde{V}
\end{array}\right](\theta)
$$

has empty singular spectrum. Let $U\left(x_{1}\right)$ be a unitary, $2 \times 2, C^{\infty}$ matrix-valued function on $\mathbb{R}$ such that $U\left(x_{1}\right)=\left[\begin{array}{ll}1 & 0 \\ 0 & 1\end{array}\right]$ if $x_{1}>1$ and $U\left(x_{1}\right)=\left[\begin{array}{rr}0 & 1 \\ -1 & 0\end{array}\right]$ if if $x_{1}<-1$ and let $U$ act on $L^{2}(C) \oplus L^{2}(C)$ in the natural manner. It is easy to show that if $\tilde{A}(\theta)=U A(\theta) U^{-1}$ then

$$
\tilde{A}(\theta)=\left[\begin{array}{cc}
-\Delta+W_{2} & 0 \\
0 & -\Delta+W_{1}
\end{array}\right]+f \cdot p+g
$$

with $f \in C_{0}^{\infty}$ and $g \in L_{0}^{\infty}$. By the last three results $\sigma_{\text {sing }}(\widetilde{A}(\theta))=\varnothing$.

\section{Appendix 1. The Twisting Trick}

The method of Theorem 6.7, adding extra degrees of freedom and then twisting, is quite powerful. In this appendix we indicate its scope of application. We use the notation from Section 6 freely. 
Proposition A.1.1. Let $V$ be a potential on $\mathbb{R}^{v}$ such that the form sum $H=-\Delta+V$ is closed and bounded from below on $Q(-\Delta) \cap Q(V)$. If $(H-i)^{-1}$ is compact then $H \in \mathscr{A}\left(a, b, \mathbb{R}^{v}\right)$ for any $(a, b)$ disjoint from $\sigma(H)$.

Proof. Condition (i) in the definition of $\mathscr{A}\left(a, b, \mathbb{R}^{v}\right)$ is true because $(H-z)^{-1}$ itself can be continued. Condition (ii) is a consequence of $p(H-i)^{-1 / 2}$ being bounded and $(H-i)^{1 / 2}(H-z)^{-1}$ having a continuation. The compactness requirements of (iii) are immediate.

Theorem A.1.2. Let $V$ be a continuous function on $\mathbb{R}$ such that $V(x)=0$ for $x<-a$ and $V(x) \rightarrow \infty$ as $x \rightarrow+\infty$. Then $-\frac{d^{2}}{d x^{2}}+V$ has empty singular continuous spectrum.

Proof. This follows the proof of Theorem 6.7 with Proposition A.1.1 replacing Theorem 6.6(c).

Theorem A.1.3. Let $V$ be a function on $\mathbb{R}^{v}$ with compact support such that $V_{+} \in L^{1}$ and $V_{-} \leqq a(-\Delta)+b$ for some $a<1$. If $H=-\Delta+V$ as a form sum then $\sigma_{\text {ess }}(H)=$ $[0, \infty), \sigma_{\text {sing }}(H)=\varnothing$ and the only possible accumulation point of the eigenvalues is 0 .

Proof. Let $A$ be the operator $\left[\begin{array}{cc}H & 0 \\ 0 & -\Delta+\alpha x^{2}\end{array}\right]$ on $L^{2}\left(\mathbb{R}^{v}\right) \oplus L^{2}\left(\mathbb{R}^{v}\right)$. Suppose that $V$ has support in the ball of radius $R$ and let $U(x)$ be a unitary, $2 \times 2, C^{\infty}$ matrix-valued function on $\mathbb{R}^{v}$ which equals $\left[\begin{array}{rr}0 & 1 \\ -1 & 0\end{array}\right]$ if $|x| \leqq R$ and $\left[\begin{array}{ll}1 & 0 \\ 0 & 1\end{array}\right]$ if $|x| \geqq R+1$. Then

$$
U A_{\alpha} U^{-1}=\left[\begin{array}{cc}
-\Delta & 0 \\
0 & -\Delta+V+\alpha x^{2}
\end{array}\right]+f \cdot p+g
$$

where $f \in C_{0}^{\infty}$ and $g \in L_{0}^{\infty}$. Since $-\Delta+\alpha x^{2}$ and $-\Delta+V+\alpha x^{2}$ have no essential spectrum, and $f \cdot p+g$ is a relatively compact perturbation

$$
\sigma_{\text {ess }}(H)=\sigma_{\text {ess }}\left(A_{\alpha}\right)=\sigma_{\text {ess }}(-\Delta) \text {. }
$$

By Theorem 6.7 and Proposition A.1.1, $\sigma_{\text {sing }}\left(A_{\alpha}\right)=\varnothing$ and the point spectrum of $A_{\alpha}$ can only accumulate at points in $\{0\} \cup \sigma\left(-\Delta+V+\alpha x^{2}\right)$. But

$$
\inf \sigma\left(-\Delta+V+\alpha x^{2}\right) \geqq \inf \sigma\left\{-(1-a) \Delta+\alpha x^{2}-b\right\}
$$

and the right-hand side goes to infinity as $\alpha \rightarrow \infty$, so zero is the only possible accumulation point of the point spectrum of $H$.

Remark. By the above one also sees that $\Omega^{ \pm}\left(U A_{\alpha} U^{-1}, A_{\alpha}^{0}\right)$ exist and are complete, where

$$
A_{\alpha}^{0}=\left[\begin{array}{cc}
-\Delta & 0 \\
0 & -\Delta+V+\alpha x^{2}
\end{array}\right] .
$$

Since $P_{\mathrm{ac}}\left(A_{\alpha}^{0}\right)=\left[\begin{array}{ll}1 & 0 \\ 0 & 0\end{array}\right]$ and 


$$
\lim _{t \rightarrow \infty}\left(U^{-1}-1\right) e^{-i t A_{x}^{0}} P_{\mathrm{ac}}\left(A_{\alpha}^{0}\right)=0
$$

we see that $\Omega^{ \pm}(H,-\Delta)$ exist and are complete. This recovers the result of $[10,14$, $32,41]$.

We next consider exterior problems, that is $H=-\Delta$ with boundary conditions on some bounded set. We begin with Dirichlet boundary conditions since these are the easiest.

Theorem A.1.4. Let $K$ be a compact set and $H$ the Dirichlet Laplacian on $L^{2}\left(\mathbb{R}^{v} \backslash K\right)$ that is the form closure of $-\Delta$ on $C_{0}^{\infty}\left(\mathbb{R}^{v} \backslash K\right)$. Then $H$ has empty singular continuous spectrum.

Proof. We first note that $H+x^{2}$ has compact resolvent and that $p\left(H+x^{2}+1\right)^{-1 / 2}$ is bounded since

$$
Q\left(H+x^{2}\right)=Q(H) \cap Q\left(x^{2}\right) \subset Q(H) \subset Q(-\Delta) .
$$

We deduce by the method of Proposition A.1.1 that $\left(H+x^{2}\right) \in \mathscr{A}\left(a, b, \mathbb{R}^{v}\right)$ for any $(a, b)$ disjoint from $\sigma\left(H+x^{2}\right)$. From this point we follow the proof of Theorem A.1.3.

Remarks. (1) As before one can prove existence and completeness of the scattering using the twisting trick.

(2) So far as we can tell Theorem A.1.4 is a new result for such general $K$, although it was proved by Lax and Phillips $[29,36]$ if $\partial K$ is smooth. It is worth observing that if one knows a priori that $\sigma_{\text {sing }}(H)$ is empty, one can short-cut some of the technicalities of the Lax-Phillips theory [36].

When one allows more general boundary conditions there is the difficulty that $p(H-z)^{-1}$ may not be bounded because functions in Dom $H$ may not be continuous on $\partial K$. It was with this difficulty in mind that we allowed general $Q$ in our definition of $\mathscr{A}(a, b, Q)$.

Theorem A.1.5. Let $K$ be a compact set in $\mathbb{R}^{v}$ and $H$ some self-adjoint extension of $-\Delta$ on $C_{0}^{\infty}\left(\mathbb{R}^{v} \backslash K\right)$. Suppose that $H+x^{2}$ has compact resolvent. Then $\sigma_{\text {ess }}(H)=$ $[0, \infty), \sigma_{\text {sing }}(H)=\varnothing$, and the only possible accumulation point of the point spectrum is 0 .

Proof. If $K \subset\{x:|x|<R\}$ we take $Q=\{x: R<|x|<R+1\}$ and follow the method of Theorem A.1.3. In order to prove that

$$
f \cdot p\left(H+x^{2}-z\right)^{-1}=(V \cdot f)\left(H+x^{2}-z\right)^{-1}+p \cdot f\left(H+x^{2}-z\right)^{-1}
$$

is well-defined and bounded we need to note that since $f \in C_{0}^{\infty}$ has support in $Q$ it follows that $f(\operatorname{Dom} H) \subset \operatorname{Dom}(-\Delta)$.

Remarks. (1) As an example if $K$ obeys the segment condition $[1,12]$ we can take Neumann boundary conditions.

(2) The twisting trick is also useful for other localisation problems. For example suppose that $K=K_{1} \cup K_{2}$ where $K_{1}$ and $K_{2}$ are disjoint compact sets and that $H, H_{1}, H_{2}$ are self-adjoint extensions of $-\Delta$ on $\mathbb{R}^{v} \backslash K, \mathbb{R}^{v} \backslash K_{1}$ and $\mathbb{R}^{v} \backslash K_{2}$ respec- 
tively with the "same boundary conditions", in the sense that if $\eta \in C_{0}^{\infty}$ with $\eta=1$ on $K_{1}$ and $\eta=0$ on $K_{2}$ then

$$
H \phi=H_{1}(\eta \phi)+H_{2}((1-\eta) \phi)
$$

where $\phi \in \operatorname{Dom} H$ if and only if $\eta \phi \in \operatorname{Dom} H_{1}$ and $(1-\eta) \phi \in \operatorname{Dom} H_{2}$. By twisting with a $U$ chosen so that

$$
U\left[\begin{array}{ll}
H+x^{2} & 0 \\
0 & -\Delta+x^{2}
\end{array}\right] U^{-1}=\left[\begin{array}{ll}
H_{1}+x^{2} & 0 \\
0 & H_{2}+x^{2}
\end{array}\right]+f \cdot p+g
$$

one may easily show that if $H_{1}+x^{2}$ and $H_{2}+x^{2}$ both have purely discrete spectrum, then so does $H+x^{2}$.

\section{Appendix 2. Measurability of Spectral Projections}

In this appendix we discuss some technical measurability questions mentioned in Sections 4 and 5. We deal throughout with a fixed measure space $M$ and a separable Hilbert space $\mathscr{H}$. A function $f: M \rightarrow \mathscr{H}$ is called measurable if $(\phi, f(m))$ is measurable for all $\phi \in \mathscr{H}$ or equivalently [34] if $f$ is measurable for the norm Borel structure of $\mathscr{H}$. A function $f: M \rightarrow \mathscr{L}(\mathscr{H})$ is called measurable if and only if $(\phi, f(m) \psi)$ is measurable for all $\phi, \psi \in \mathscr{H}$, or equivalently if $f(m) \psi$ is measurable for all $\psi$; this is not the same as norm measurability since $\mathscr{L}(\mathscr{H})$ is not separable. By writing

$$
(\phi, f(m) g(m) \psi)=\sum_{n}\left(\phi, f(m) \eta_{n}\right)\left(\eta_{n}, g(m) \psi\right)
$$

where $\eta_{n}$ is an orthonormal basis of $\mathscr{H}$, we see that products of measurable operator-valued functions are measurable. A map $f$ from $M$ to the unbounded self-adjoint operators is called measurable if any of the conditions of the following lemma are satisfied.

Lemma A.2.1. If $f$ is a map from $M$ to the unbounded self-adjoint operators on $\mathscr{H}$, the following conditions are equivalent:

(i) $(f(m)+z)^{-1}$ is measurable for all $z \notin \mathbb{R}$

(ii) $e^{i t f(m)}$ is measurable for all $t \in \mathbb{R}$

(iii) the spectral projection $E_{I}(f(m))$ of $f(m)$ is measurable for all intervals $I$.

Proof. These are all based on explicit limits. For example to prove (i) implies (iii) we use

$$
E_{[a, b]}(A)=\lim _{r \rightarrow \infty} \lim _{n \rightarrow \infty} \frac{1}{\pi} \int_{a-(1 / r)}^{b+(1 / r)}\left[\left(A-\lambda-\frac{i}{n}\right)^{-1}-\left(A-\lambda+\frac{i}{n}\right)^{-1}\right] d \lambda .
$$

Proposition A.2.2. If $A(m)$ is a measurable family of self-adjoint operators on $\mathscr{H}$, then the spectral projections $P_{\mathrm{pp}}(A(m))$ are measurable.

Proof. Let $\tilde{\mathscr{H}}$ be the Hilbert space of Hilbert-Schmidt operators on $\mathscr{H}$ and let $\tilde{A}(m)=\operatorname{Ad} A(m)$ so that

$$
e^{i A(m) t}(X)=e^{i A(m) t} X e^{-i A(m) t}
$$


and by Proposition A.2.1, $\tilde{A}(m)$ is measurable, as is its spectral projection $E_{\{0\}}(\tilde{A}(m))$. Since $B=B^{*} \in \operatorname{Ran} E_{\{0\}}(\tilde{A}(m))$ if and only if its spectral projections commute with those of $A(m)$, we see that

$$
\operatorname{Ran} P_{\mathrm{pp}}(A(m))=\overline{\cup\left\{\operatorname{Ran} B: B \in \operatorname{Ran} E_{\{0\}}(\tilde{A}(m))\right\}} .
$$

Thus if $B_{r}$ is a countable dense set in $\tilde{\mathscr{H}}$ and $\psi_{n}$ is a countable dense set in $\mathscr{H}$ then

$$
\operatorname{Ran} P_{\mathrm{pp}}(A(m))=\overline{\left\{\phi_{r n}(m)\right\}}
$$

where

$$
\phi_{r n}(m)=\left[E_{\{0\}}(\tilde{A}(m)) B_{r}\right] \psi_{n}
$$

is a countable set of measurable functions. Applying the Gram-Schmidt perocess to reconstruct the projection we see that $P_{\mathrm{pp}}(A(m))$ is measurable.

\section{References}

1. Agmon, S. : Lectures on elliptic boundary value problems. Princeton, London, Toronto: Van Nostrand 1965

2. Agmon, S. : Spectral properties of Schrödinger operators and scattering theory. Ann. Scuola Norm. Sup. Pisa Sci. II 2, 151-218 (1975)

3. Alsholm, P. K., Kato, T. : Scattering with long range potentials. Proc. Symp. Pure Math. 23, 393-399 (1973)

4. Amrein, W., Georgescu, V.: On the characterization of bound states and scattering states in quantum mechanics. Helv. Phys. Acta 46, 635-658 (1973)

5. Amrein, W., Martin, P., Misra, B. : On the asymptotic condition in scattering theory. Helv. Phys. Acta 43, 313-344 (1970)

6. Avron, J., Herbst, I. : Spectral and scattering theory of Schrödinger operators related to the Stark effect. Commun. math. Phys. 52, 239-254 (1977)

7. Birman, M. S., Solomjak, M. Z. : On estimates of singular numbers of integral operators III. Vestn. Leningr. Univ. 24, 35-48 (1969)

8. Coddington, E., Levinson, N. : Theory of ordinary differential equations. New York: McGrawHill 1955

9. Combes, J. M., : An algebraic approach to scattering theory. (unpublished) (1970)

10. Combescure, M., Ginibre, J. : Scattering and local absorption for the Schrödinger operator. J. Funct. Anal. (to appear)

11. Davies, E. B. : Scattering from infinite sheets. Math. Proc. Camb. Phil. Soc. 82, 327-334 (1977)

12. Deift, P. : Classical scattering theory with a trace condition. Princeton: Princeton University Press 1978

13. Deift, P. : Application of a commutator formula. Duke Math. J. (to appear)

14. Deift, P., Simon, B. : On the decoupling of finite singularities from the question of asymptotic completeness. J. Funct. Anal. 23, 218-238 (1976)

15. Deift, P., Simon, B. : A time dependent approach to the completeness of multiparticle quantum systems. Comm. Pure Appl. Math. 30, 573-583 (1977)

16. Deift, P., Trubowitz, E. : Inverse scattering on the line. Comm. Pure Appl. Math. (to appear)

17. Dinaburg, E., Sinai, Ya. : The one dimensional Schrödinger equation with a quasiperiodic potential. Funct. Anal. Appl. 9, 8-21 (1975)

18. Dollard, J. : On the definition of scattering subspaces in non-relativistic quantum mechanics. J. Math. Phys. 18, 229-232 (1977)

19. Eastham, M. S. P.: The spectral theory of periodic differential equations. Scottish Academic Press 1973

20. Enss V. : A note on Hunziker's theorem. Commun. math. Phys. 52, 233-238 (1977) 
21. Enss, V.: Asymptotic completeness for quantum mechanical potential scattering. Commun. math. Phys. 61, 258-291 (1978)

22. Gross, H., Grümm, H. R., Narnhofer, H., Thirring, W. : Algebraic theory of Coulomb scattering. Acta Phys. Austr. 40, 97-103 (1974)

23. Hepp, K. : Scattering theory in the Heisenberg ferromagnet. Phys. Rev. 135, 95-97 (1972)

24. Hörmander, L. : The existence of wave operators in scattering theory. Math. Z. 146, 69-91 (1976)

25. Jost. R. : The general theory of quantized fields. Providence, RI: American Mathematical Society 1965

26. Klein, O. : Die Reflexion von Elektronen an einem Potentialsprung nach der relativistischen Dynamik von Dirac. Z. Physik 53, 157-165 (1929)

27. Kuroda, S. : Scattering theory for differential operators I. J. Math. Soc. Japan 25, $75-104$ (1973)

28. Lavine, R. B. : Scattering theory for long range potentials. J. Funct. Anal. 5, 368-382 (1970)

29. Lax, P., Phillips, R. S. : Scattering theory. New York, London: Academic Press 1967

30. Levitan, B., Sargstan, I.: Introduction to spectral theory. Am. Math. Soc. Monograph. Transl. 39, 1975

31. Magnus, W., Winkler, S. : Hill's equation. New York: Wiley 1966

32. Pearson, D. : General theory of potential scattering with absorption at local singularities. Helv. Phys. Acta 47, 249--264 (1974)

33. Pearson, D. : A generalization of the Birman trace theorem. J. Funct. Anal. 28, 182-186 (1978)

34. Reed, M., Simon, B. : Methods of modern mathematical physics. I. Functional analysis. New York, London: Academic Press 1972

35. Reed M., Simon, B. : Methods of modern mathematical physics. II. Fourier analysis. New York, London: Academic Press 1975

36. Reed, M., Simon, B. : Methods of modern mathematical physics. III. Scattering theory. New York, London: Academic Press 1979

37. Reed, M., Simon, B. : Methods of modern mathematical physics. IV. Analysis of operators. New York, London: Academic Press 1978

38. Ruelle, D. : A remark on bound states in potential scattering theory. Nuovo Cimento 61 A, 655-662 (1969)

39. Ruelle, D. : On the asymptotic condition in quantum field theory. Helv. Phys. Acta 35, 147-163 (1962)

40. Ruijenaars, S., Bongaarts, P. : Scattering theory for one-dimensional step potentials. Ann. Inst. Henri Poincaré 26A, 1-17 (1977)

41. Semenov, Yu. : Wave operators for the Schrödinger equation with strongly singular short range potentials. Lett. Math. Phys. 1, 457-462 (1977)

42. Simon, B. : Lectures on trace ideal methods. In: London Mathematical Society Lecture Notes. Cambridge: Cambridge University Press 1979

43. Simon, B.: Geometric methods in multiparticle quantum systems. Commun. math. Phys. 55, 259-274 (1977)

44. Simon, B. : N-body scattering in the two cluster region. Commun. math. Phys. 58, 205-210 (1978)

45. Sinha, K. : On the absolutely and singularly continuous subspace in scattering theory. Ann. Inst. Henri Poincaré 26A, 263-277 (1977)

46. Streater, R. F. : Spin wave scattering. In: Scattering theory in mathematical physics, $273-298$. Dordrecht, Stuttgart: Reidel 1974

47. Wilcox, C. : Scattering states and wave operators in the abstract theory of scattering. J. Funct. Anal. 12, 257-274 (1973)

Communicated by J. Ginibre

Received May 31, 1978 
